\title{
Protocolo de atención a niños y adolescentes con cardiopatía congénita en odontopediatría. Revision bibliográfica.
}

\section{Resumen}

Introducción. Las cardiopatías congénitas son las malformaciones más habituales en el desarrollo de los niños los cuales, además, son susceptibles a desarrollar infecciones sistémicas microbianas secundarias a infecciones locales polimicrobianas como consecuencia de procedimientos dentales invasivos o toman fármacos, por ejemplo anti-coagulantes, antiarrítmicos, u otros, que pueden interferir con el manejo odontológico. Objetivo. Proponer un protocolo de manejo odontológico que permita detectar las cardiopatías congénitas o ya identificadas tener un abordaje dental que disminuya el riesgo de sepsis o endocarditis para el paciente pediátrico. Método. Revisión de la literatura a través de artículos indexados en Cochrane, Medline, Lilacs, EMBASE, Amedeo y SciELO, enfatizando los últimos cinco años, en los idiomas: francés, italiano, portugués, inglés y español. Resultados. Se presenta un protocolo
Juan Manuel Cortes-Ramírez', Carmen de la Luz Ayala-Escandón ${ }^{1}$, Juan Manuel de Jesús Cortes de la Torre², Raúl Arturo Cortes de la Torre, Laura Otilia Salazar", Alfredo Salazar de Santiago ${ }^{1}$, Celia Elizabeth Luna Pacheco'.

de abordaje odontológico que disminuya los factores de riesgo ante la presencia de cardiopatías congénitas. Conclusión. Ya que existe una au-sencia de información sobre la relación de las cardiopatías congénitas y la odontología infantil, por ello la importancia de contar con un protocolo para el tratamiento de pacientes pediátricos mejorando su atención, pronóstico, calidad de vida y disminuyendo los factores de riesgo ante procedimientos dentales invasivos.

Palabras clave: Cardiopatías congénitas; Odontopediatra; niños; Protocolo de atención.

Artigo de Revisão

\section{Protocolo de atendimento para crianças e adolescentes com doença cardíaca congênita em odontopediatria. Revisão da literatura.}

\section{Resumo}

Introdução. As cardiopatías congênitassão as malformações mais comuns no desenvolvimento das crianças, os quais são também mais suscetíveis a desenvolver infecções microbianas sistêmicas secundárias ou infecções locais polimicrobbianaas como consequência de procedimentos odon-

\footnotetext{
${ }^{1}$ Área de ciencias de la salud, Universidad Autónoma de Zacatecas, México

${ }^{2}$ Residente de cardiología del Instituto Nacional de Cardiología "Ignacio Chávez", México.

${ }^{3}$ Escuela de Medicina "Ignacio Santos", Tecnológico de Monterrey, México

${ }^{4}$ Universidad Autónoma de Nuevo León, México.
} 
tológicos invasivos ou tomam drogas como antiarrítmicos, anticoagulantes dentre outras, que podem interferir no tratamento odontológica. Objetivo. Propor um protocolo de atendimento odontológico, permitindo detectar cardiopatia congênita ou já identificadas para adotar uma abordagem odontológica que diminua o risco de sepse ou endocardite para o paciente pediátrico. Método. Revisão da literatura através de artigos indexados no Cochrane, Medline, Lilacs, EMBASE, Amedeo e SciELO, enfatizando os últimos cinco anos, nos idiomas: francês, italiano, português, inglês e espanhol. Resultados. Apresenta-se um protocolo para abordagem odontológica que reduz os fatores de risco na presença de doença cardíaca congênita. Conclusão. Como há falta de informação sobre a relação de cardiopatias congênitas e Odontopediatria, portanto, a importância de ter um protocolo para o tratamento de pacientes pediátricos para melhorar seus cuidados, o prognóstico, a qualidade de vida e diminuindo os fatores de risco antes de procedimentos odontológicos invasivos.

Palavras-chave: Doença cardíaca congênita; odon-topediatra; Protocolo de cuidados.

\section{Pediatric dental care protocol for children with heart disease. Literature review.}

\section{Abstract}

Introduction. Congenital heart diseases are the most common childhood development malformations. These children are predisposed to developing infective endocarditis caused by bacteremia due to dental procedures. Objective. The propose of this review is to establish a protocol for dental management of children with congenital heart disease, minimizing risks caused by dental treatment. Methods: A review of the literature was conducted searching articles indexed in Cochrane, Medline, Lilacs, EMBASE, Amedeo and SciELO, emphasizing the past five years, in languages: French, Italian, Portuguese, English and Spanish. Results. A protocol for dental approach is presented, which reduces risk factors in the presence of congenital heart disease. Conclusion. There is a scarse information regarding the relationship between pathology and dentistry, therefore the importance of having a protocol for the treatment of these patients that improve their quality of life and decrease the risk factors.

Key words: Congenital heart disease (MeSH); Pediatric Dentistry (MeSH); Children (MeSH); CARE Protocol (MeSH).

\section{Introducción}

En el mundo nacen cada año 135 millones de niños, de los cuales 1 de cada 33 recién nacidos vivos se ve afectado por una anomalía congénita, entre 4 y 12 por cada 1000 (1-3), que generan a su vez 3,2 millones de discapacidades al año. ${ }^{4}$ 
La designación de "cardiopatías congénitas" (CC) es un término general que se usa para describir anomalías del corazón y los grandes vasos, que están presentes desde el momento del nacimiento. Se usa para describir lesiones anatómicas de una o varias de las cuatro cámaras cardiacas (malformación de Ebstein), de los tabiques que las separan, (comunicación interventricular o interauricular) o de las válvulas o tractos de salida (estenosis pulmonar o aortica), que están presentes desde el momento del nacimiento. La mayor parte se debe a una embriogénesis defectuosa de una estructura normal, o a un fallo de dicha estructura para establecer los cambios que deben ocurrir en la transición de la vida intrauterina a la extrauterina (conducto arterioso persistente). Aunque algunas son incompatibles con la vida, muchas permiten al enfermo llegar a la edad adulta, e incluso algunas pasan desapercibidas a lo largo de toda su vida, todo depende de la repercusión hemodinámica. ${ }^{5}$ Dos tercios de estos enfermos morían dentro del primer año de vida, sin un diagnóstico oportuno y sin tratamiento establecido. Actualmente un número considerable de niños alcanza la edad adulta a consecuencia de un tratamiento adecuado, médico quirúrgico o mixto, o porque el defecto provoca sólo mínimas alteraciones hemodinámicas. En los últimos cincuenta años se ha avanzado en el manejo de estas enfermedades, lo que ha disminuido la mortalidad y mejorado la calidad de vida de los niños afectados. Motivo por el cual persisten presentándose y estar preparados para saber qué hacer en el momento en que los encontremos. ${ }^{5}$

Las CC son de las más frecuentes malformaciones congénitas y representan alrededor del $30 \%$ de ellas, con una incidencia de entre
6-8/1000 nacido vivo. Muchas de ellas se encuentran presentes desde el periodo prenatal y su detección han permitido intervenciones en el feto, mejorando el pronóstico. Son más comunes en prematuros y en abortos. Las más habituales de presentación son: la comunicación interventricular (CIV), el conducto arterioso permeable (PCA o ductus persistente), la comunicación interauricular (CIA), la tetralogía de Fallot (TF) la estenosis pulmonar y la estenosis aórtica. En México, son la tercera causa de muerte en niños preescolares de un año y la sexta en niños de tres años. ${ }^{5}$

Su etiología es desconocida; en más del 90\% se relaciona con algunos factores genéticos mendeliano o multifactorial. Las aberraciones cromosómicas son responsables de menos del $10 \%$ de los casos. Las enfermedades genéticas que se relacionan son el síndrome de Down, Turner (45, XO), trisomías 13 (Patau), 15, 17 y 18, síndrome de Hurler y otras mucopolisacaridosis, síndrome de Kartagener (hay situs inversus), síndrome de Ehlers-Danlos y Marfan, Osler-Weber-Rendu, enfermedad de Crouzon, síndrome de Apert, síndrome de Noonan, síndrome de Di George, homocistinuria y la glucogenosis tipo II. ${ }^{6}$

La incidencia aumenta de 2 a 10 veces en los hijos de un progenitor afectado, o en los hermanos de un sujeto con CC. Dentro de los factores ambientales se reconoce al virus de la rubéola (infección in útero durante el primer trimestre del embarazo), las radiaciones, agentes químicos (alcohol, tintes de cabello), fármacos (DFH, talidomida, litio, ácido retinoico), tabaquismo, drogadicción. ${ }^{7,} 8$ Un defecto en un gen puede causar distintas cardiopatías, y la misma cardiopatía puede deberse a 
defectos en diferentes genes. Asimismo, una malformación puede ser causada por la mutación de un gen, pero también por microdeleciones en distintos loci. En la herencia mendeliana clásica el fenotipo se expresa como consecuencia de mutaciones en uno (dominante) o ambos (re-cesiva) alelos de un gen. Aunque la lista de síndromes con afectación cardiaca debidos a herencia mendeliana por mutación de un gen es muy larga, la proporción de casos debidos a este tipo de herencia es baja, estimándose en $5-10 \%$. Algunas miocardiopatias se deben a mutaciones en el ADN mitocondrial, heredándose exclusivamente de la madre. ${ }^{8}$

Las CC pueden clasificarse de diversas formas como cianógenas, (cortocircuito de derecha a izquierda) y acianógenas (cortocircuito de izquierda a derecha) más constantes las acianógenas, el 90 \%, (CIA, CIV, PCA), de las cianógenas, la más común es la tetralogía de Fallot. Con flujo pulmonar normal, alto y disminuido. En simples (por ejemplo, CIA) o complejas (CIA más coartación de aorta), si se presentan defectos aislados o asociados. Con repercusión hemodinámica y sin ella. Existe la clasificación secuencial que divide al corazón en 3 segmentos: visceroatrial, atrioventricular y ventriculoarterial. Estos tres segmentos están conectados uno al otro de una manera secuencial, independientemente de la relación espacial que guarden entre sí. ${ }^{9-11}$

Las manifestaciones clínicas dependen del tipo de CC, pueden encontrarse: disnea, taquicardia, taquipnea, cianosis de la piel y de las mucosas, policitemia, síntomas cerebrales (mareo o síncope), facies rubicunda, acropaquias (dedos hipocráticos o en palillo de tambor, soplos y retraso en crecimiento y desarrollo). ${ }^{11-16}$
Las manifestaciones bucales encontradas en pacientes con CC dependerán de los defectos congénitos. Se puede observar globalmente: un color rojo azulado de la mucosa bucal y de la piel dela cara por policitemia y por cianosis. Petequias, púrpuras y/o hematomas por trombocitopenia. Entre los hallazgos de laboratorio se encuentran concentraciones altas de hemoglobina y hematocritos, trom-bocitopenia, disminución en las cantidades de fibrinógeno y cambios en los tiempos de coagulación. ${ }^{16}$

Las manifestaciones dentales más frecuentes encontradas en pacientes con cardiopatías congénitas son: cianosis de mucosas, que se presenta principalmente en las encías, en las mucosas alveolares y en el paladar, y se debe a la deficiencia de oxígeno en la sangre, por lo que ésta toma una coloración azulada; retraso en la erupción dental, el cual es proporcional al retraso en el desarrollo físico del paciente; hipoplasia del esmalte; alteraciones en la posición normal de los dientes; dientes color blanco-azulado o blanquecino; vasodilatación pulpar manifiesta; aumento de la incidencia de caries dental; y enfermedad periodontal por mala higiene bucal. ${ }^{17,18}$

La caries es una enfermedad infecciosa multifactorial, en la que interviene, entre otros factores, una microbiota muy variada, las bacterias más frecuentes son los estreptococos del grupo mutans, seguidas del género de Lactobacillus, estas bacterias intervienen en la formación de la biopelícula, pero con una composición diferente según la localización. Ya antes de la erupción dentaria el 50\% de los bebes, presentan una colonización bucal por Streptoccocus mutans. ${ }^{19}$ Los Streptococos del grupo mutans (S. mutans, S. sobrinus, S. cricetus, S. ratius, S. ferus, S. downwi y $S$. macacae), son 
los más importantes en la etiopatogenia de la caries, por lo que la prescripción de un tratamiento antibacteriano, en pacientes de alto riesgo que aún no tengan lesión evidente, es aconsejable de modo profiláctico. ${ }^{20}$

Las infecciones sistémicas microbianas son las que asientan en pacientes con susceptibilidad de infectarse con mayor facilidad por bac-terias capsuladas del género Streptococcus o Haemophilus, $\mathrm{o}$ en pacientes con alteraciones generalizadas del sistema inmune que faciliten una septicemia. Generalmente, el primer paso suele ser la bacteriemia, que se produce tras un procedimiento invasivo o cruento, como los practicados en odontología. Es necesario un control odontológico preventivo permanente mediante higiene bucal, educación dietética y fluoraciones periódicas, para mantener sus bocas sanas, evitando la atención de urgencia previa a intervenciones dentales, que en sí mismas son complejas. ${ }^{21}$

Al acudir a consulta odontológica la mayoría de los pacientes ya han sido diagnosticados y se encuentran en tratamiento y control por el cardiólogo. Es posible que se presente un caso el cual no ha sido diagnosticado, y que durante la anamnesis y la exploración física, el odontólogo, sea capaz de identificar los signos y síntomas sugestivos, y ante la sospecha, enviarlo con el especialista para valoración y confirmación o no de la CC.

\section{Protocolo de manejo.}

1.-En casos sintomáticos, remitir al cardiólogo.

2.-Preguntar los medicamentos que está tomando y ver si hay interacción con los que el odontopediatra utiliza, como anestésicos locales con y $\sin$ vasoconstrictor.
Tabla 1. Tabla adaptada de: Gutiérrez JL, Bagán J, Bascones A, Llamas R, Llena J, Morales A, Noguerol $B$, Planells P, Prieto J, Salmerón JI. Documento de consenso sobre la utilización de profilaxis antibiótica en cirugía y procedimientos dentales. Med Oral Patol Oral Cir Bucal 2006; 11(2): 188-205.

\begin{tabular}{|c|c|c|}
\hline Procedimiento & $\begin{array}{c}\text { Alto } \\
\text { Riesgo }\end{array}$ & $\begin{array}{c}\text { Bajo } \\
\text { Riesgo }\end{array}$ \\
\hline Anestesia intraligamentosa & $X$ & \\
\hline Anestesia troncular & $X$ & \\
\hline $\begin{array}{c}\text { Aparatología removible } \\
\text { ortopédica }\end{array}$ & & $X$ \\
\hline Biopsias & $X$ & \\
\hline $\begin{array}{l}\text { Colocación de bandas } \\
\text { para aparatología fija }\end{array}$ & & $X$ \\
\hline $\begin{array}{l}\text { Colocación de } \\
\text { hilo retractor }\end{array}$ & & $x$ \\
\hline $\begin{array}{l}\text { Colocación y retiro } \\
\text { de sutura quirúrgica }\end{array}$ & $x$ & \\
\hline $\begin{array}{c}\text { Colocación de coronas, cuñas } \\
\text { y banda matriz }\end{array}$ & & \\
\hline Cirugía oncológica maxilar & $x$ & \\
\hline Cirugía Ortognatica & $x$ & \\
\hline Extracciones & $x$ & \\
\hline $\begin{array}{c}\text { Incisiones } \\
\text { para drenado }\end{array}$ & $x$ & \\
\hline Profilaxis periodontal & $x$ & \\
\hline $\begin{array}{c}\text { Endodoncias, Pulpotomía y } \\
\text { pulpectomía }\end{array}$ & $X$ & \\
\hline $\begin{array}{c}\text { Reimplantes dentarios trau- } \\
\text { máticos }\end{array}$ & $x$ & \\
\hline Toma de radiografías & & $x$ \\
\hline Toma de impresiones & & $x$ \\
\hline
\end{tabular}


3.-Ver si existe tendencia hemorrágica por uso de anticoagulantes y tener cuidados técnicos por la tendencia hemorrágica aumentada.

4.-En los casos que exista indicación, como en todos los procedimientos dentales que involucre unan manipulación del tejido gingival, de la región periapical o perforaciones a la mucosa oral, $^{22}$ (Tabla 1) antibioterapia profiláctica por riesgo de endocarditis o endarteritis infecciosa.

Factores de riesgo para endocarditis infecciosa: Tradicionales: Malformaciones congénitas, fiebre reumática y otras disfunciones valvulares adquiridas, miocardiopatía hipertrófica, prolapso mitral con regurgitación.

- De máximo riesgo: Endocarditis previa, prótesis valvulares biológicas o mecánicas, cardiopatía congénita cianótica compleja.

- Discutidas. Paciente con marcapaso, des-fibrilador, catéteres intravasculares, con derivación ventrículo yugular (por hidrocefalia), injertos vasculares sintéticos, pacientes en hemodiálisis.

- Bajo o nulo. Comunicación interauricular aislada; seis meses después de un cierre de CIV, CIA o PCA; trasplante cardiaco, pacientes con fiebre reumática previa sin cardiopatía y abuso de drogas por vía parenteral.

- Definitivos. Procedimientos dentales que pro-vocan hemorragia gingival y de las mucosas, tonsilectomía y/o adenoidectomía, cirugía en mucosa respiratoria o intestinal, cistoscopia, cateterismo ureteral o cirugía del tracto urinario si hay infección, endodoncia, endoprótesis aórticas.
- Inciertos. Pacientes sometidos a tratamiento dental por incisión alveolar sin ulceraciones preexistentes, colocación de coronas o puentes dentales, septoplastía nasal, ecocardiografía transesofágica. ${ }^{8-16}$

\section{La American Heart Association (AHA),} preocupados por mantener y controlar el índice de incidencia de CC, norma en 1997 una nueva evaluación del protocolo de recomendaciones para la prevención de infecciones sistémicas bacterianas basado en el tratamiento preventivo con antibióticos en pacientes considerados de alto riesgo con la indicación de intervenciones clínicas o quirúrgicas que pueden provocar una bacteriemia transitoria. ${ }^{24}$ La utilización de profilaxis antibiótica en los procedimientos y técnicas quirúrgicas en odontopediatría, dependerá del tipo de procedimiento a realizar. Laprofilaxissebasaenel riesgodel procedimiento y evitar una bacteriemia tras infección focal ${ }^{25}$ en este tipo de pacientes que presentan riesgo de endocarditis infecciosa, con el conocimiento de la actividad de los antimicrobianos frente a los odontopatogenos. ${ }^{26}$ La selección idónea del antibiótico dependerá del tipo de procedimiento siguiendo los mismos principios que para el adulto. Teniendo en cuenta únicamente las peculiaridades de farmacocinética y toxicidad, por ello algunos antibióticos como las quinolonas no están recomendadas en pacientes pediátricos. Del mismo modo las tetraciclinas no deberán administrase en menores de 8 años, ${ }^{27}$ y la selección debe cumplir con las siguientes características:

+ Espectro bacteriano adecuado, cubriendo todas las especies involucradas en las infecciones polimicrobianas locales o monomicrobianas focales distales, incluyendo microorganismos aerobios, microaerófilos, sin olvidar los 
anaerobios que debido a la dificultad en su aislamiento en ocasiones no son considerados prevalentes en bacteriemias de origen oral.

+ Espectro clínico amplio, para cubrir el mayor número de procedimientos odontológicos.

+ Espectro ecológico restringido para limitar al máximo los efectos sobre la flora saprófita habitual.

+ Farmacocinética y farmacodinamia adecuadas, para permitir su utilización en monodosis pre-operatoria en el caso de la profilaxis, o intervalos de dosificación amplios en el tratamiento preventivo de corta duración, con vidas medias o formulaciones de liberación prolongada que mantengan concentraciones adecuadas a nivel local (fluido gingival) o sistémico (suero) durante todo el tiempo que dure el procedimiento odontológico (profilaxis).

+ Perfil de seguridad adecuado.

Los antibióticos administrados oralmente que son efectivos ante infecciones odontogénicas incluyen penicilina, clindamicina, eritromicina, cefadroxilo, metronidazol y las tetraciclinas. Penicilina $\mathrm{V}$ fue la penicilina de elección ante infecciones odontogénicas. Es bactericida, y aunque su espectro de acción, es relativamente estrecho, era el apropiado para los tratamientos

\begin{tabular}{|c|c|c|}
\hline \multicolumn{3}{|c|}{ Profilaxis para procedimientos dentales, cavidad oral, tracto respiratorio o esofágico } \\
\hline Situación & Agente & Régimen \\
\hline Profilaxis estándar & Amoxicilina & niños $50 \mathrm{mg} / \mathrm{Kg}$ oral, 1 hora antes del procedimiento \\
\hline Intolerancia a la vía oral & Ampicilina & $\begin{array}{l}\text { IM o IV niños } 50 \mathrm{mg} / \mathrm{Kg} \text { en } 30 \text { minutos antes del } \\
\text { procedimiento }\end{array}$ \\
\hline \multirow{4}{*}{ Alérgicos a penicilina } & Clíndamicina & Oral, niños $20 \mathrm{mg} / \mathrm{Kg}$, 1 hora antes del procedimiento \\
\hline & Cefalexina & Oral, niños $50 \mathrm{mg} / \mathrm{Kg}$, 1 hora antes del procedimiento \\
\hline & Azitromicin & Oral, niños $15 \mathrm{mg} / \mathrm{Kg} 1$ hora antes del procedimiento \\
\hline & Claritromicina & Oral niños $15 \mathrm{mg} / \mathrm{Kg} 1$ hora antes del procedimiento \\
\hline \multirow{2}{*}{$\begin{array}{l}\text { Alérgicos a penicilina e } \\
\text { intolerancia a la vía oral }\end{array}$} & Clindamicina & IV niños $20 \mathrm{mg} / \mathrm{Kg}$, 30 minutos antes del procedimiento \\
\hline & Cefazolina & $\begin{array}{l}\text { IM o IV niños } 25 \mathrm{mg} / \mathrm{Kg} \text {, } 30 \text { minutos antes del } \\
\text { procedimiento }\end{array}$ \\
\hline
\end{tabular}

Tabla 2. Esquema de antibioticoterapia profiláctica para endocarditis en pacientes pediátricos que recibirán tratamiento dental. Tabla adaptada de: Wilson W y Cols $2007^{22}$ 
de infecciones odontogénicas. ${ }^{23}$

Para la profilaxis de endocarditis asociada con tratamientos dentales, se sugiere la siguiente de acuerdo a la American Heart Association.22 (Tabla 2)

5- Es necesaria la eliminación de focos sépticos, las infecciones dentales y los tratamientos endodónticosque pueden provocarendocarditis infecciosa, endarteritis infecciosa, abscesos cerebrales (en malformaciones vasculares cerebrales). El uso de antisépticos tópicos en la cavidad oral reduce el inóculo bacteriano, pero no ha demostrado ser eficaz en la profilaxis de la colonización bacteriana. No obstante, el uso de antisépticos preoperatorios en la cavidad oral puede reducir las complicaciones derivadas del trauma en la mucosa, especialmente en pacientes inmunodeprimidos $\mathrm{y}$ en pacientes con mala higiene oral. ${ }^{28}$

6- De primera intención contraindicada la anestesia general.

Las técnicas específicas y necesarias para el diagnóstico de las alteraciones cardiovasculares deben formar parte del conocimiento del Odontopediatra y como tratarlas. Para ello se debe recalcar que el trabajo interdisciplinario es la premisa en equipo de profesionales que se dedican al cuidado de la salud de sus pacientes, por lo que cualquier profesional de la salud, y esto incluye al odontólogo infantil necesita contar con un protocolo de atención. Saber llevar a cabo una amnesis rigurosa principalmente en pacientes con CC. Detectar el nivel de riesgo del desarrollo de una endocarditis bacteriana y de ser así informar al medico cardiólogo que lo asiste para obtener información más precisa de su situación actual, dado que la instrumentación de las intervenciones dentales invasivas deben ser reforzadas. Así como la prevención de farmacoterapia recomendada deben ser extremadas antes de los procedimientos que puedan encaminar a una situación de bacteriemia, específicamente en aquellos pacientes con historia previa de la enfermedad o portadores de prótesis valvular cardiaca.

El desarrollo de resistencia por parte de los microorganismos al antimicrobiano, algunas incógnitas sobre la eficacia de los tratamientos preventivos, posibles reacciones adversas $\mathrm{o}$ toxicas a los antibióticos, da lugar a preguntas sobre el riesgo-beneficio de su prescripción. La Academia Americana de Odontología Pediátrica recomienda el uso conservador de los antibióticos ante el incremento de resistencias, por lo que se requieren de estudios multicentricos para conocer la evolución de éstas, y que sean útiles al momento de la decisión, evitando la proliferación de tales resistencias. $^{29}$

\section{Conclusiones}

Al revisar la literatura existe escasa información sobre la relación de las cardiopatías congénitas y la odontología infantil que establezcan protocolos para el tratamiento de estos pacientes pediátricos. Se propone un protocolo de manejo odontopediatrico y que todo odontólogo, previo a cualquier procedimiento bucal, deba elaborar una historia clínica, que permita detectar las CC y tomar las precauciones necesarias. Previo a cualquier tratamiento dental a pacientes infantiles con CC, con algún síndrome o alteración que resulte no ser conocida, es recomendable primeramente ponerse en contacto con el médico tratante, a fin de determinar la susceptibilidad previa a infecciones inducidas por bacteriemia; disminuyendo así, los factores de riesgo ante procedimientos dentales invasivos. 


\section{Referencias bibliográficas}

1 Cloarec S, Magontier N, Vaillant MC, Paillet C, Chantepie A. Prévalence et répartion des cardiopathies congénitales en Indre et Loire. Évaluation du diagnostic anténatal (1991-1994). Arch Pediart 1999; 6: 1059-65.

2 Manetti A, Pollini I, Cecchi F, De Simone L, Cianciulli D, Carbone C, Romanelli A, Bianchi F, Dolara A. Epidemiologia delle malformazioni cardiovascolari. III. Prevalenza e decorso in 46895 nati vivi alla maternita di Careggi, Firenze nel periodo 19751984. G Ital Cardiol 1993; 23: 145-52.

3 Zaqout M, Aslem ES, Oweida FS, De Wolf D. Prevalence of congenital heart disease among Palestinian children born in the Gaza Strip. Cardiol Young 2014; 24(05): 905-09.

4 Sandoval N. Cardiopatías congénitas en Colombia y en el mundo. Rev Colomb Cardiol 2015; 22(1): 1-2.

5 García R, Sotelo JI, Rodríguez A. (2015). Embriología del aparato Cardiovascular. En Cortes Ramírez JM, et alt. (Edit.), Cardiología 2015 (pp. 116-119). Zacatecas: Editorial de la Universidad Autónoma de Zacatecas.

6 Gelb BD. History of Our Understanding of the Causes of Congenital Heart Disease. Circ Cardiovasc Genet 2015; 8(3): 529-36. 7 Uribe AK, Díaz-Vélez C, Cerrón-Rivera C. Características epidemiológicas y clínicas de las cardiopatías congénitas en menores de 5 años del Hospital Almanzor Aguinaga Asenjo: Enero-Diciembre 2012. Horiz Med 2015; 15(1):49-56.

8 Pawluk MS, Campaña H, Gili JA, Comas B, Giménez LG, Villalba MI, López JS; Scala S. Poletta F. Deteminantes sociales adversos y riesgo para anomalías congénitas seleccionadas. Arch Argent Pediatr 2014; 112(3): 215-23.

9 Martínez-Quintana E, Romero-Requejo A, Rodríguez-González F. Cardiopatías congénitas y embarazo. Clínica e Investigación en Ginecología y Obstetricia 2015; 42(3):

10 Sánchez-Urbina R, Galaviz-Hernández C, Sierra-Ramírez A, Moran-Barroso VF, García-Cavazos R. Trascendencia de los factores ambientales y genéticos en cardiopatías congénitas el caso de la enzima MTHFR. Perinatol Reprod Hum 2006; 20(1-3): 39-47.

11 Calderón J, Cervantes-Salazar JL, Curi-Curi PJ, Ramírez Marroquín S. Problemática de las cardiopatías congénitas en México. Arch Cardiol Mex. 2010; 80(2):133-40.

12 Baltaxe E, Zarante I. Prevalencia de malformaciones cardíacas congénitas en 44.985 nacimientos en Colombia. Arch Cardiol Mex 2006; 76(4): 264.

13 Vilas LT, Albernaz EP, Costa R. Prevalence of congenital heart defects in patients with Down syndrome in the municipality of Pelotas. Braz J Pediatr 2009; 85(5): 403-7.

14 Palmero MI, Briceño J, Mendoza M, Bhuedo C. Cardiopatía congénita en recién nacido de madre VIH (+): A propósito de un caso. Rev Obstet Ginecol Venez 2006; 66(1): 39-42.

15 Ayala J. Cardiología para pediatras de atención primaria. Rev Pediatr Aten Primaria 2009; 11(Supl 17): 451-56.

16 Marín V, Rosati P, Las Heras MS, Rivera C, Castillo C. Hypercaloric diet and nutritional recovery in infants with congenital heart disease. Rev Chil Pediatr 1990; 61(6): 305.

$17 \mathrm{Liu}$, Zifeng, et al. Counseling role of primary care physicians in preventing early childhood caries in children with congenital heart disease. Int J Environ Res Public Health 2014; 11(12): 12716-25.

18 Pimentel Elizangela Lins Cavalcanti, Azevedo VM, de Almeida Lima CR, Reis Luciana Carvalho, Andrea De L. Caries experience in young children with congenital heart disease in a developing country. Braz Oral Res 2013; 27(2): 103-108.

19 Kim Seow, W. Environmental, maternal, and child factors which contribute to early childhood caries: A unifying conceptual model. Intl J Paed Dent 2012; 22(3): 157-68.

20 Gutiérrez JL, Bagán J, Bascones A, Llamas R, Llena J, Morales A, Noguerol B, Planells P, Prieto J, Salmerón JI.

Documento de consenso sobre la utilización de profilaxis antibiótica en cirugía y procedimientos dentales. 
Med Oral Patol Oral Cir Bucal 2006; 11(2): 188-205. Balejo R, Porto S, Cavalca S. Bacteriemia em pacientes periodontais: revisão de literatura. Braz J Periodontol 2014; 24(4): 29-40.

22 Wilson W, Taubert KA, Gewitz M, Lockhart PB, Baddour LM, Levison M, Bolger A, Cabell CH, Takahashi M, Baltimore RS, Newburger JW, Strom BL, Tani LY, Gerber M, Bonow RO, Pallasch T, Shulman ST, Rowley AH, Burns JC, Gardner T, Durack D. Prevention of Infective Endocarditis Guidelines From the American Heart Association: A Guideline From the American Heart Association Rheumatic Fever, Endocarditis, and Kawasaki Disease Committee, Council on Cardiovascular Disease in the Young, and the Council on Clinical Cardiology, Council on Cardiovascular Surgery and Anesthesia, and the Quality of Care and Outcomes Research Interdisciplinary Working Group. Circulation 2007; 116(15): 1736-54.

23 Moss AJ, Adams FH, Emmanovilides GC, editors. Heart disease in infants, children adolescents. 3a ed. Baltimore: Williams \& Wilkins; 1983.

24 Nakano K, Ooshima T. Common knowledge regarding prevention of infective endocarditis among general dentists in Japan. J. Cardiol. 2010; 12(2): 31-5.

25 Bayer AS, Ward JI, Ginzton LE, Shapiro SM. Evaluation of New Clinical criteria for the diagnosis of infective endocarditis. Am J Med 1994; 96: 211-19.

26 Robertson-Malt S, Afrane B, Elbarbary M. Prophylactic steroids for pediatric open heart surgery. Cochrane Database of Systematic Reviews 2007; [access 23 of July 2015] Issue 4. Art. No.: disponible en: http://www.cochrane.org/CD005550/VASC_ prophylactic-steroids-for-pediatric-open-heart-surgery. CD005550. DOI: 10.1002/14651858.CD005550.pub2.

27 Bascones A, Aguirre JM, Bermejo A, Blanco A, Gay-Escoda C, González MA, Gutiérrez JL,Jiménez Y, Liébana J, LópezMarcos JF, Maestre JR, Perea EJ, Prieto J, Vicente JC. Documento de consenso sobre el tratamiento antimicrobiano de las infecciones bacterianas odontogénicas. Av Odontoestomatol 2005; 21(6): 311-9.

28 Barroso MG, Cortela D, Mota WP. Endocardite bacteriana: da boca ao coração. Ciên Est Acad Med 2014; 1(2): $47-57$. 29 American Academy on Pediatric Dentistry Clinical Affairs Committee; American Academy on Pediatric Dentistry Council on Clinical Affairs. Guideline on antibiotic prophylaxis for dental patients at risk for infection. Revised 2014. Pediatr Dent 2015; 36(6):287-92.

Recibido: 01-10-15

Aceptado: 21-10-15

Correspondencia autor: MgSc Carmen de la Luz Ayala Escandón Calle 1º de Mayo No. 426-3,

Centro Histórico Zacatecas, Zacatecas, C.P. 98000, México, Telefono: +52 4929250940 E-mail: claescandon@yahoo.com 